\title{
Pulmonary Gangrene due to a Septic Emboli in a Young Female
}

\author{
S Grewal ${ }^{1 *}$, Hannah Knox ${ }^{1}$, Henry Kim², Jericho Hallare ${ }^{2}$, Shirley Kim², Timothy Law ${ }^{2}$, Leonard \\ Ranasinghe $^{3}$ and Sameir Alhadi ${ }^{4}$ \\ ${ }^{1}$ Internal Medicine Residency, Saint Agnes Medical Center, USA \\ ${ }^{2}$ Medical Student, California Northstate University College of Medicine, USA \\ ${ }^{3}$ Faculty of Medicine, California Northstate University College of Medicine, USA \\ ${ }^{4}$ Emergency Medicine, Saint Agnes Medical Center, USA
}

*Corresponding author: Sarbjot Grewal, Internal Medicine Residency, Saint Agnes Medical Center, Fresno, CA, 8112 East Central Avenue, Del Rey, CA 93616, USA

To Cite This Article: S Grewal. Pulmonary Gangrene due to a Septic Emboli in a Young Female. 2020 - 9(3). AJBSR.MS.ID.001391. DOI: 10.34297/ AJBSR.2020.09.001391.

Received: 䡅: June 16, 2020; Published: 眥 June 30, 2020

\begin{abstract}
Rationale: A rare case of necrotizing pneumonia secondary to septic DVT and use of ECMO and lung transplant in refractory respiratory failure.

Objectives: To evaluate the role of ECMO and lung transplant in necrotizing pneumonia.

Methods: We present a case report of a young female with Methicillin resistant necrotizing pneumonia, secondary to a septic deep venous thrombosis.

Measurements: none

Main results: ECMO may be employed in younger, healthy patients with limited comorbidities as in our patient.

Conclusions: We present a successful ECMO application in a COVID -19 positive patient with severe ARDS

Keywords: Pulmonary Gangrene, Septic Emboli, ECMO, Necrotizing Pneumonia, MRSA, ARDS, Lung Transplant

Abbreviations: PMHX: Past Medical History; MRI: Magnetic Resonance Imaging; PE: Pulmonary Embolism; DVT: Deep Vein Thrombosis; MRSA: Methicillin-Resistant Sathylococcus Aureus; CXR: Chest X-Ray; FiO2: Fractional Inspiration of Oxygen; ECMO: Extracorporeal Membrane Oxygenation; VVECMO: venous-venous Extracorporeal Membrane Oxygenation; VA ECMO: venous-Arterial Extracorporeal Membrane; CCRT: Continuous Renal Replacement Therapy
\end{abstract}

\section{Introduction}

18 year old non-smoker female from California with pmhx of asthma presented with right knee pain and shortness of breath. On admission, vitals were stable, saturating well on room air. MRI of knee confirmed parietal gastrocnemius tear along with some hyperintensity of bone marrow of femur. Chest X-ray on admission revealed bilateral multifocal opacities. Upon admission, influenza and coccidioides serology were negative. Ultrasound doppler of the right lower extremities revealed deep vein thrombosis. Computerized tomography angiogram chest revealed subsegmental pulmonary embolism within the right lower lobe, with bilateral opacities. Heparin drip for management for acute PE and DVT. Due to decreasing oxygen saturation to $93 \%$, the patient required $4 \mathrm{~L} /$ min via nasal cannula.

Patient continued to be hypoxic and hypotensive, underwent emergent intubation. Blood cultures returned positive growing Methicillin-resistant Staphylococcus Aureus (MRSA). Repeat CXR showed bilateral pneumothoraces requiring bilateral chest tube placements.

Patient's hospital stay was further complicated by heparin induced thrombocytopenia, transitioned to argatroban drip. Due to 
continuing worsening oxygenation and increasing FiO2 requirement of (1), the patient was eventually started on veno-venous ECMO (VV-ECMO) via right Internal jugular Avalon cannula on day 5. Next day, due to hemodynamic deterioration, veno-arterial ECMO (VA-ECMO) was initiated. The patient continued to require FiO2 of [1] via ECMO and FiO2 of 0.4 via pressure controlled mechanical ventilation. Thoracic washouts were done to minimize risk of infection, in addition to appropriate infectious control measures.

Due to the irreversible pulmonary gangrene developed, the patient's lungs were deemed unviable, thus was evaluated for lung transplant for a better outcome. Patient was transferred to a higher level of care facility where she will receive VA-ECMO, CRRT and be evaluated for lung transplant. At the time of writing of this report, the patient is still pending lung transplant.

\section{Materials and Methods}

Not a study, $\mathrm{n} / \mathrm{a}$

\section{Results and Discussion}

Necrotizing pneumonia is a highly lethal and rapidly progressive disease affecting young adults. $1-10 \%$ can be attributed to Staphylococcus aureus as the pathologic organism [2]. About $0.8-7 \%$ of all community-acquired pneumonia cases, can manifest as necrotizing pneumonia [3]. Some studies correlate necrotizing pneumonia to a primary viral illness, such as influenza virus, with superimposed bacterial infection [2]. The likely source in this patient is the soft tissue injury, given that three other team members endorsed similar injuries, which were infected with MRSA.

A common presentation in the general population is a viral infection followed by a superimposed MRSA infection. Given that the patient was a softball player, and recently multiple players were diagnosed with MRSA infections, we propose a case of a primary septic deep venous thrombosis, leading to seeding pulmonary emboli, with resultant MRSA necrotizing pneumonia. With the bilateral multifocal nature of lung injury, this was the result of septic emboli, primarily from infected DVT as evident on MRI initially.

$21 \%$ of patients with necrotizing pneumonia develop pneumothoraces, The high prevalence of pneumothoraces in the setting of necrotizing pneumonia can be attributed to barotrauma due to high level of ventilator pressure settings in combination with the underlying pathology of lung necrosis and development of cavitations. For patients who develop refractory hypoxemic failure, VV-ECMO has shown promising results, followed by surgical resection [4].

ECMO serves as a cardiopulmonary support, and should betimely employed in appropriate candidates. Indications of employment include prolonged stay in intensive care units due to refractory respiratory failure, may be used as a bridge for both cardiac and lung transplant and support for lung resections in hemodynamically unstable patients. ECMO should be used as a supportive therapy rather than a disease modifying treatment [5]. Contraindications of ECMO include severe chronic organ dysfunction, advanced age, high mortality rate, disseminated malignancy, cardiac arrest. In cases of VA ECMO, systemic thromboembolism is commonly reported. Heparin induced thrombocytopenia may occur in patients receiving ECMO due to heparin use as seen in our patient and upon detection, heparin must be discontinued [5].

\section{Conclusion}

This case demonstrates a rare manifestation of MRSA induced necrotizing pneumonia due to a septic DVT, resulting in pulmonary gangrene. This case highlights the importance of maintaining suspicion for rare complications in young hypoxic patients. Furthermore, it emphasizes good outcomes regarding appropriate use of ECMO in young patients with limited comorbidities and severe acute hypoxemic respiratory failure.

\section{Conflict of Interest}

No conflicts of Interest

\section{Reference}

1. National Ambulatory Medical Care Survey (NAMCS) and National Hospital Ambulatory Medical Care Survey (NHAMCS) 2009 - 2010.

2. Kreienbuehl L, Charbonney E, Eggimann P (2011) Community-acquired necrotizing pneumonia due to methicillin-sensitive Staphylococcus aureus secreting Panton-Valentine leukocidin: a review of case reports. Ann Intensive Care 1(1): 52.

3. Masters IB, Isles AF, Grimwood K (2017) Necrotizing pneumonia: An emerging problem in children. Pneumonia (Nathan) 9: 11.

4. Merlo A, Reid T, Burks AC, Long Jason (2019) Venovenous Extracorporeal Membrane Oxygenation and Pulmonary Resection for Necrotizing Pneumonia. Ann Thorac Surg 107(2): 115-117.

5. Makdisi G, Wang IW (2015) Extra Corporeal Membrane Oxygenation (ECMO) review of a lifesaving Technology. J Thorac Dis 7(7): 166-176. 